\title{
Evidence for quantum melting in the two-dimensional electron system on a thin helium film
}

\author{
T. Günzler, B. Bitnar, G. Mistura *, S. Neser, P. Leiderer \\ Fakul:äc für Physik, Ciniversity of Konstanz, P.O. Box 5560M684, D-78464 Konstanz, Germany.
}

Received 13 June 1995; accepted for publication 2 August 1995

\begin{abstract}
The real and imaginary parts of the dielectric response of surface state electrons (SSE) on belum films adsorbed on oxidized Si platelets have been measured with a microwave carity at $10 \mathrm{GHz}$. Preliminary measurements taken at $T=1.2 \mathrm{~K}$ show an abrupt increase of the SSE mobility at electron densities near $10^{11} \mathrm{~cm}^{-2}$. which is suggestive of quantum melting of the Wigner solid. Reproducibility of this effect on different Si wafers is discussed.
\end{abstract}

\section{Introduction}

The phase diagram of the two-dimensional electron system (2DES) at low temperature $T$ is characterized by a freezing of the Coulomb gas to a Wigner solid at low electron density $n$, where electrons behave classically, and, more interestingly, by a quantum melting of the Wigner solid to a degenerate Fermi liquid at high $\pi$. The classical part of the 2DES phase diagram, in particular the Wigner crystallization, has been investigated using SSE on the surface of liquid helium, an almost ideal substrate owing to its pure and predictable experimental conditions [1]. However, an instability of the charged surface limits the accessible range of electron density to $n=2 \times 10^{9} \mathrm{~cm}^{-2}$. A thin heliurn film is instead additionally stabilized by van der Waals forces which allow the film to reach higher $n$ and thus permit study of the influence of the Fermi energy and possibly the quantum

* Correspording a uthor. Fax: + 497531883127 :

e-mail: giampaolo.mistura@uni-konstanzde. melting [2]. So far only the reverse isansition from a degenerate Fermi gas to a Wigner solid has been observed in semiconductor heterostruccures using extremely high magnetic fields.

On an helium film, the direc Coulomb repulsion is weakened by the screening due to the substrate which leads to a shrinking of the solid phase in the $(n, T)$ phase diagram. In particular, the quantum region of the phase diagram is pushed to lower electron densities [2]. This reduction increases as the film thickness decreases Etz et al. [3] demonstrated that it is possible to charge helium films to electron densities up to $n \approx 10^{11} \mathrm{~cm}^{-2}$, and found that at such $n$ the film thickness is greatly reduced and is essentially determined by the large electrostatic pressure. The reduction of the film thickness during charging (at $n \geq 10^{10} \mathrm{~cm}^{-2}$ ) has the effect of increasing the probability that the SSE will tunnel through the film at some rough spot on the surface. On an insularing substrate, those electrons which pass through the helium film stick to the surface, prevent other electrons from tunneling and become scattering and pinning centers for the 
movement of the SSE on the film. Furthermore, as the film gets thinner, the lateral movement of the SSE becomes increasingly perturbed by the roughness of the underlying substrate and by the presence of surface impurities. This is the cause of the progressive disappearance of the signal in lowfrequency conductivity measurements as the electron density approaches $10^{11} \mathrm{~cm}^{-2}$. To avoid this complication, we have measured the response of the SSE at microwave frequencies.

\section{Experimental}

The experimental setup consists of a cylindrical cavity $19.5 \mathrm{~mm}$ in diameter and $20 \mathrm{~mm}$ in length, forming a microwave resonator which is excited in the fundamental $\mathrm{TM}_{010}$ mode at a frequency $f \approx 10 \mathrm{GHz}$. A rectangular $(30 \mathrm{~mm} \times 10 \mathrm{~mm})$ oxjdized silicon platelet, which serves as a substrate for the helium film, is placed along the axis of the cavity, parallel to the high frequency electric field of this mode. The cavity is inserted in a closed copper cell immersed in the liquid helium of a glass dewar. During the measurements, high-purity helium gas is condensed into the sample cell at 1.2 $\mathrm{K}$. The level of liquid helium inside the cavity with respect to the top face of the $\mathrm{SiO}_{2}$ can be adjusted using the frequency shift of the cavity itself as a level meter. The helium film is charged by firing a small filament mounted at the top of the cavity every $\approx 35$ and by increasing very slowly the clamping voltage $U$, applied berween the isolated Si platelet and the grounded cavity, in order to guarantee a saturated charging of the film. During the charging, the resonance frequency $f$ of the cavity and the transmitted power $P$ are recorded by a computer. From $P$ and $f$ we then calculate the dimensionless relative absorption $\alpha$ and the effective susceptibility $\%$ of the SSE: which represent the imaginary and real components, respectively; of the dielectric function $\tilde{\epsilon}(\omega, k)$ of the SSE at fixed $\omega$ and $k$.

\section{Results and discussion}

As substrates for the helium films we have so far sudied platelets cut from thernally oxidized
p-Si wafers with $\mathrm{SiO}_{2}$ thicknesses $d_{\mathrm{s}}$ of 2600 and $4900 \AA$ (Sil), and from a sputtered p-Si wafer $d_{\mathrm{s}} \approx 5000 \AA$ (Sill). Fig. 1 shows the results taken on Sil plated with a $400 \AA$ thick helıum film, plotted against $U$. Assuming that the film is charged at saturation, $n=\epsilon_{s} \epsilon_{0} U ; d_{s} e$, where $\epsilon_{\mathrm{s}} \approx 4$ is the permittivity of $\mathrm{SiO}_{2}$. For the daca presented here $n \approx 4 \times 10^{10} \mathrm{~cm}^{-2}$ for $U=1 \mathrm{~V}$. As the electron density $n$ is increased from $n=0$, we find three prominent structures appearing in both $x$ and $\gamma$. First, at low $n, \alpha$ and $\%$ are seen to vary linearly with $n$, although with slopes of opposite sign. This is the expected behavior for non-interacting electrons. Secoodly, around $U=0.3 \mathrm{~V}$, both curves bend over 10 a region with a much smaller slope, implying a reduction in the SSE mobility. We interpret this kink as the transition from the classical Coulomb gas to a Wigner solid pinned to the surface. Its location $\left(n \approx 1.3 \times 10^{10} \mathrm{~cm}^{-2}\right)$ is much higher than the value of $\sim 2.5 \times 10^{9} \mathrm{~cm}^{-2}$ obtained by extrapolating the experimental phase diagram on bulk belium to $1.2 \mathrm{~K}$. This is due to shielding effects caused by the finite polarizability of the $\mathrm{SiO}_{2}$ layer and Fermi energy corrections. The

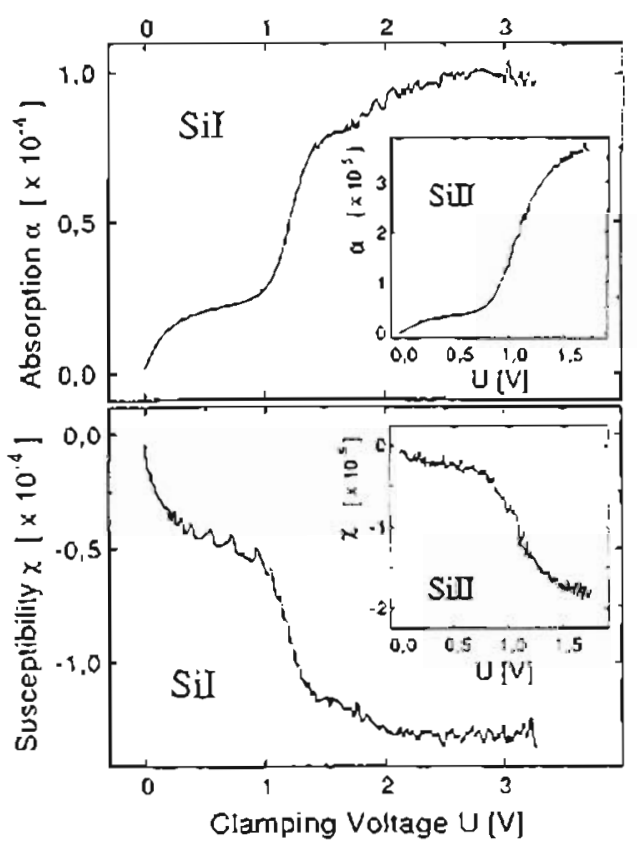

Fig 1. Absorption $z$ (top) and susceptibility $\%$ (botton) of the SSE taken on SiI and on Sill (insert) as functions of the clamp. ing voltage. Intial helium film thickness $\approx 400 \AA$. See text for derails. 
rounding of this transition is probably related to inhomogeneities of the $\mathrm{SiO}_{2}$ surface. Similar behavior bas been found in low-frequency conductivity measurements of SSE on different substrates [4]. Finally, at $U$ around $I V, \alpha$ and $\chi$ display a second step, such that the SSE mobility appears to increase again. Note that the signals after the step assume values close to the linear extrapolation of the data at low $U$. This, together witb the fact that the feature occurs at a value of $n$ which is expected from theoretical arguments [2], is suggestive of a quantum melting of the electron system from a Wigner solid to a degenerate Fermi gas.

This result cannot be explained as a plasmon resonance of the free SSE, because for our cavity, it is expected to occur at a density $n \approx 3 \times 10^{13} \mathrm{~cm}^{-2}$ : 700 times more than we observe. Furchermore, this step cannot be due to charge carriers inside the Si substrate. The $\mathrm{p}$-Si, together with the $\mathrm{SiO}_{2}$ layer, forms a MOSFET-like struccure with the electrons on top of the He film as the gate electrode. Owing to the strong clamping field. a 2D hole gas (2DHG) develops in the lowest sublevel of the inversion layer at the interface between $\mathrm{Si}$ and $\mathrm{SiO}_{2}$. This $2 \mathrm{DHG}$, of the same density as the $2 \mathrm{DEG}$ on top of the $\mathrm{He}$ film, also gives rise to microwave absorpion. However, in the range of $U^{\prime}$ values used in our measurements, the contribution of the $2 D H G$, as well as that of the charge carriers in the bulk si, is quite small. To prove that this is indeed the case, we evacuated the sample cell so that only a few monolayers of He covered the Si platelet. and repeated the charging procedure. The result is displayed in Fig. 2. The larger noise is due to temperature fluctuations in the cavity after each firing of the filament, which are now much larger than before due to the absence of a superfluid film. Both curves, $x$ and $\%$ are straight lives parallel to the horizontal axis up to $U=10 \mathrm{~V}$. This means that neither the electrons which are strongly bound to the $\mathrm{SiO}_{2}$ surface nor; more importantly, the charge carriers inside the Si. give rise to any appreciable contribution to the microwave signal in our measurements. After this dry charging, we iepeated the measurements with 2 saturated helium film (see Fig. 2). The new curves are similar to those of Fig. 1, but with two important differences: (1) the voltage scale is shifted to

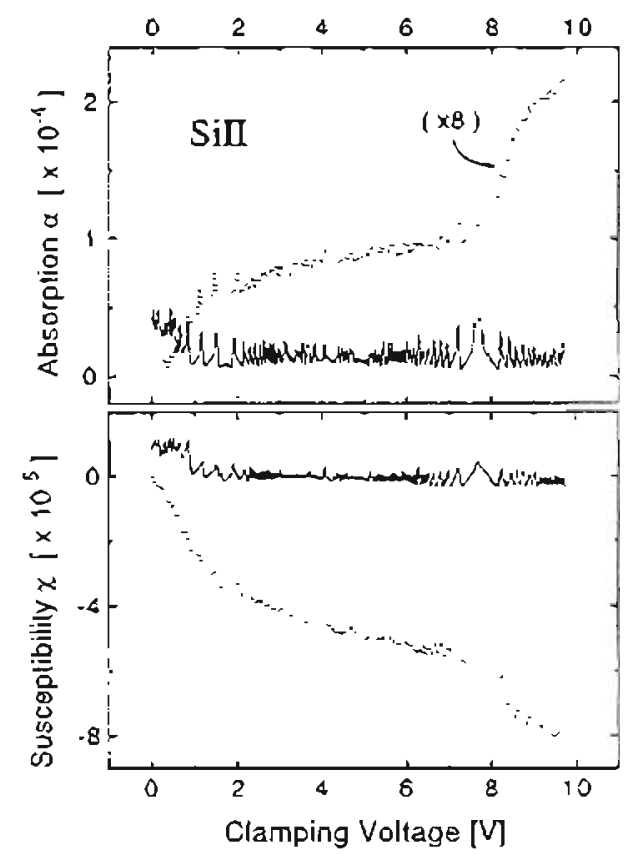

Fic. 2. Absorption $x$ (10p) and susceptibility $\times$ (bottom) of the SSE as functions of the clamping voltage measured on a "dry" $\mathrm{SiO}_{2}$ suriace (contunuous line) and on a $400 \AA$ thick helium flon. right after the "dry" charging (dotted linc).

higher values to overcome the repulsive potential due to the electrons pinned to the $\mathrm{SiO}_{2}$, and (ii) the overall change in $\alpha$ is much smaller, implying a lower mobility of the SSE, probably caused by: scattering with an increased number of surface defecis.

Fig. 1 also shows curves iaken on SiII of similar shapes to those on Sil. It is interesting to note that compared to SiI, the changes in $\alpha$ and $\%$ are about two times and eight times smaller, respectively'. These differences can be explained in terms of a lower mobility on Sill, a result expected due to the surface of the sputtered Si. which is obviously not as smooth as that of SiI. Besides this, the second step occurs at a different roltage. Similar shifts have also been found with the same sample after several charging runs or after thermal cycling to room temperature. In other words, the quantitative reproducibility of the data so far seems 10 depend very much on the "history" of the sample. Systematic measurements are now in progress to better understand these effects. The next goal is to map out the phase diagram. 


\section{Acknowledgements}

This research has been partially supported by the HCM-network of the European Community, contract No. ERBCHRXCT930374. Some of the Si samples used were kindly provided by Professor Jutzi.

\section{References}

[1] See for example, F.I.B. Williams, Surf. Sci. $113(1982) 371$. and reiereaces therein.
[2] F.M. Peeters and P.M. Platzman. Phys. Rev. Lett. 50(1983) 2021;

F.M. Peeters, Phys. Rev. B 30 (1984) 159;

M. Saitoh, Surf. Sci. 229 (1990) 356.

[3] H. Etz, W. Gombert, W' Idstein and P. Leiderer, Phys. Rev. Let. 53 (1984) 2567.

[4] K. Kajita, J. Phys. Soc. Jpn. 54 (1985) 4092;

H.W. Jiang and A.J. Dahm, Phys. Rev. Lett. 62 (1989) 1396 : F. Mugele, U. Albrecht, P. Leiderer and K. Kono, J. Low Temp. Phys. 89 (1992)? 73. 